\title{
Identification of Quinoline-Based RIP2 Kinase Inhibitors with an Improved Therapeutic Index to the hERG Ion Channel
}

Pamela A. Haile, ${ }^{*}{ }^{\dagger} \odot$ Linda N. Casillas, ${ }^{\dagger}$ Michael J. Bury, ${ }^{\dagger}$ John F. Mehlmann, ${ }^{\dagger}$ Robert Singhaus, Jr., ${ }^{\dagger}$ Adam K. Charnley, ${ }^{\dagger}$ Terry V. Hughes, ${ }^{\dagger}$ Michael P. DeMartino, ${ }^{\dagger}$ Gren Z. Wang, ${ }^{\dagger}$ Joseph J. Romano, ${ }^{\dagger}$ Xiaoyang Dong, ${ }^{\dagger}$ Nikolay V. Plotnikov, ${ }^{\dagger}$ Ami S. Lakdawala, ${ }^{\dagger}$ Maire A. Convery, ${ }^{\dagger}$ Bartholomew J. Votta, ${ }^{\dagger}$ David B. Lipshutz, ${ }^{\dagger}$ Biva M. Desai, ${ }^{\dagger}$ Barbara Swift, ${ }^{\dagger}$ Carol A. Capriotti, ${ }^{\dagger}$ Scott B. Berger, ${ }^{\dagger}$ Mukesh K. Mahajan, ${ }^{\dagger}$ Michael A. Reilly, ${ }^{\dagger}$ Elizabeth J. Rivera, ${ }^{\dagger}$ Helen H. Sun, ${ }^{\dagger}$ Rakesh Nagilla, $^{\dagger}$ Carol LePage, ${ }^{\dagger}$ Michael T. Ouellette, ${ }^{\dagger}$ Rachel D. Totoritis, ${ }^{\dagger}$ Brian T. Donovan, ${ }^{\dagger}$ Barry S. Brown, ${ }^{\dagger}$ Khuram W. Chaudhary, ${ }^{\dagger}$ Peter J. Gough, ${ }^{\dagger}$ John Bertin, ${ }^{\dagger}$ and Robert W. Marquis ${ }^{\dagger}$

${ }^{\dagger}$ GlaxoSmithKline, Collegeville Road, Collegeville, Pennsylvania 19426, United States

‡GlaxoSmithKline, Gunnels Wood Road, Stevenage, Hertfordshire SG1 2NY, United Kingdom

Supporting Information

ABSTRACT: RIP2 kinase was recently identified as a therapeutic target for a variety of autoimmune diseases. We have reported previously a selective 4-aminoquinoline-based RIP2 inhibitor GSK583 and demonstrated its effectiveness in blocking downstream NOD2 signaling in cellular models, rodent in vivo models, and human ex vivo disease models. While this tool compound was valuable in validating the biological pathway, it suffered from activity at the hERG ion channel and a poor $\mathrm{PK} / \mathrm{PD}$ profile thereby limiting progression of this analog. Herein, we detail our efforts to improve both this off-target liability as well as the PK/PD profile of this series of inhibitors through modulation of lipophilicity and strengthening hinge binding ability. These efforts have led to inhibitor 7, which possesses high binding affinity for the ATP pocket of RIP2 $\left(\mathrm{IC}_{50}=1 \mathrm{nM}\right)$ and inhibition of downstream cytokine production in human whole blood $\left(\mathrm{IC}_{50}=10 \mathrm{nM}\right)$ with reduced hERG activity $(14 \mu \mathrm{M})$.

KEYWORDS: RIP2, NOD1, NOD2, kinase inhibitor, hERG

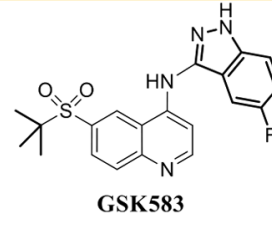

RIP2K binding $\mathrm{IC}_{50}$ $5 \mathrm{nM}$ human whole blood $\mathrm{IC}_{50} \quad 234 \mathrm{nM}$ hERG IC I0

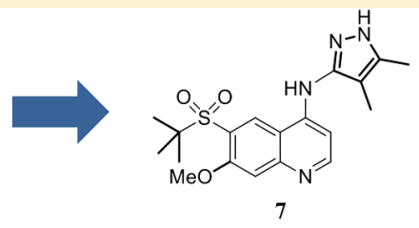

RIP2K binding $\mathrm{IC}_{50} \quad 1 \mathrm{nM}$ human whole blood $\mathrm{IC}_{50} \quad 10 \mathrm{nM}$ hERG IC $_{50} \quad 14.5 \mathrm{uM}$
$\mathrm{P}$ attern recognition receptors (PRRs) are the key sentinels that drive the innate immune system's response to invading pathogens. While NOD-like receptors NOD1 and NOD2 play a central role in identifying and mounting a response to bacterial invasion, dysregulated signaling of these receptors have been implicated in a variety of inflammatory diseases. ${ }^{1-5}$ Proximal to NOD1 and 2 is receptor-interacting-protein 2 kinase (RIP2 kinase), which drives downstream signal transduction following the activation of either NOD1 or NOD2. We and others have demonstrated that a RIP2 kinase inhibitor is effective in blocking signaling downstream of NOD1 and NOD2. ${ }^{6-11}$ We've also demonstrated that RIP2 kinase inhibition is effective in modulating spontaneous inflammatory responses in human disease biopsy tissues obtained from both Crohn's and ulcerative colitis patients illustrating the potential for RIP2 kinase inhibitors in modulating human inflammatory diseases. It is also interesting to note that stimulation of either NOD1 or NOD2 with their respective agonists, i.e., DAP or MDP, can act synergistically with TLR agonists to produce enhanced cytokine production, suggesting that a RIP2 kinase inhibitor may have far broader therapeutic utility than anticipated based on this receptor crosstalk or synergy. ${ }^{12,13}$ These data suggest that targeting the NOD1/NOD2 pathway with a small molecule inhibitor may have broad application in the treatment of a wide variety of inflammatory diseases.

Because of the well-recognized role that both NOD1 and NOD2 play in innate immune signaling, proinflammatory cytokine production, and pathogenesis of inflammation, a variety of approaches to inhibit this pathway have been explored. Early attempts focusing on the direct inhibition of NOD1 and/or NOD2 have not been successful in producing viable drug candidates for a variety of reasons, most notably selectivity, overall compound developability, and an inability to assay a compound's direct binding to either NOD1 or NOD2. ${ }^{14}$ Recently a variety of groups have explored the inhibition of the more tractable downstream kinase RIP2, a protein at which both NOD1 and NOD2 signaling converge. Not unexpectedly, it was recognized that early kinase inhibitors such as the epidermal

Received: July 27, 2018

Accepted: September 11, 2018

Published: September 26, 2018 
growth factor tyrosine kinase inhibitor gefitinib as well as the p38 inhibitor SB203580 were also potent inhibitors of RIP2 kinase activity. ${ }^{15,16}$ Recent reports have detailed structure-based approaches toward the identification of RIP2 kinase inhibitors. These compounds are generally somewhat more selective than those detailed above but still lack the requisite level of kinase activity that would permit accurate interpretation of biological phenotypes as well as the ability to progress these compounds on to more detailed biological characterization, safety assessment investigations, and, ultimately, man.

We have reported recently the identification of GSK583, a potent and selective inhibitor of RIP2 kinase (Figure 1) that was

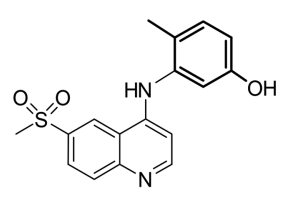

1

$\mathrm{MW}=328 ; \operatorname{cog} \mathrm{P}=3.1, \mathrm{LipE}=5.4$

Human Whole Blood $\mathrm{IC}_{50}=90 \mathrm{nM}$ Poor kinase selectivity

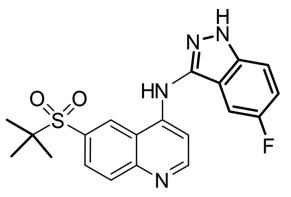

GSK583

$M W=398 ; \operatorname{cog} \mathrm{P}=4.5, \mathrm{LipE}=3.8$

Human Whole Blood $\mathrm{IC}_{50}=237 \mathrm{nM}$ Excellent kinase selectivity hERG IC 50 $_{50}=7.5 \mathrm{uM}$
Figure 1. RIP2 kinase screening hit 1 and the selective inhibitor GSK583.

derived from high-throughput screening hit $1 .^{6}$ Analog 1 was shown to be a potent inhibitor of RIP2 but lacked the requisite kinase selectivity that would allow for progression into in vivo models. Structure-activity relationship (SAR) studies aimed at modifications of the back pocket binding group of 1 led to the identification of GSK583. GSK583 exhibited an excellent kinase selectivity profile as well as the ability to modulate inflammatory responses in both murine in vivo models and ex vivo human disease biopsy tissues obtained from both Crohn's and ulcerative colitis patients. Despite these promising properties GSK583 was relegated to a tool compound as further development was limited due to a combination of potency versus the hERG ion channel and a less than optimal pharmacokinetic profile. This combination led to a PK/PD profile that afforded, at best, a modest therapeutic window between this off-target interaction relative to the human whole blood assay $\mathrm{IC}_{50}(237 \mathrm{nM})$. Ideally a progressable drug candidate would possess a greater than 100fold window between the free $C_{\max }$ at a targeted $\mathrm{IC}_{90}$ of RIP2 kinase inhibition at $24 \mathrm{~h}$ versus $\mathrm{hERG}$ related $\mathrm{QT}$ prolongations in a rabbit ventricular wedge model. ${ }^{17}$ This Letter details efforts to increase overall inhibitor efficacy and therapeutic windows by simultaneously reducing the off-target interactions with the hERG ion channel, increasing activity in the human whole blood assay, and optimizing the overall pharmacokinetic profile of this inhibitor template.

The deleterious effects of excess lipophilicity in compound design has been well documented. With this in mind, it was rationalized that a significant portion of the lipophilic nature of GSK583 was imparted by the indazole back pocket moiety. This, in combination with the basicity of the quinoline N1 nitrogen, were likely responsible for both the interaction of GSK583 with the hERG channel as well as the suboptimal pharmacokinetic profile. Indeed, general strategies for reducing hERG activity include reducing lipophilicity, lowering the $\mathrm{p} K_{\mathrm{a}}$ of basic nitrogens, as well as the elimination of aromatic groups. ${ }^{18,19}$ Extensive SAR studies within the 4-aminoquinoline series of RIP2 kinase inhibitors revealed that attempts to reduce the basicity of the $\mathrm{N} 1$ nitrogen of the 4-aminoquinoline core were not well tolerated as this modification weakened the critical hinge binding interaction between Met98 and the quinazoline template thereby reducing overall inhibitor affinity. Based on this data a strategy to reduce both the lipophilicity and aromaticity of GSK583 was pursued. As a surrogate ahead of assessing our targeted drug levels against QT prolongation in more complex models, we targeted compounds with a > 100-fold window between potency in human whole blood and activity in the hERG Q-patch electrophysiology assay. In addition to improving the window to hERG activity in vitro, it was anticipated that this strategy would also have the beneficial effect of reducing potential metabolic liabilities associated with GSK583, thereby improving its overall pharmacokinetic profile and efficacy further widening the therapeutic window in vivo.

As previously disclosed, the X-ray co-crystal structure of GSK583 bound within the ATP binding pocket of RIP2 kinase revealed a critical hydrogen bonding interaction between $\mathrm{N} 1$ of the indazole and Asp164 of the protein (Figure 2). Additionally,

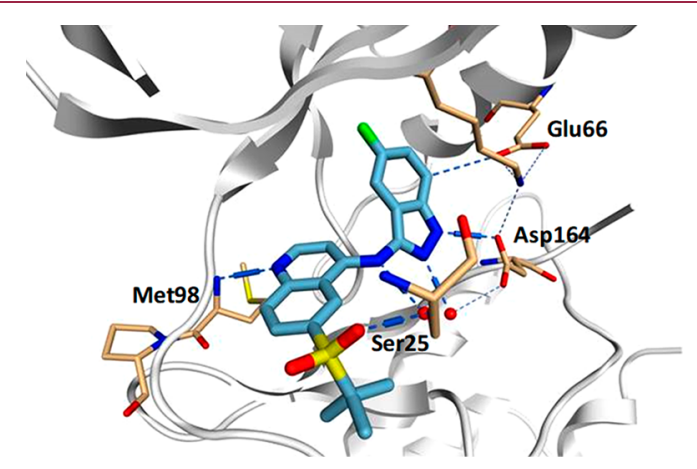

Figure 2. X-ray co-crystal structure of RIP2 kinase inhibitor GSK583.

several weaker interactions with the indazole were observed: proton- $\pi$ interactions with Lys 47 and a nontraditional hydrogen bond of the hydrogen at the C7 position with Glu66 (2.5 $))^{6,20}$ These data suggested that modifications that maintained the key hydrogen bonding interaction with Asp 164 while attempting to remove the lipophilic aromatic moiety of the indazole may reduce overall inhibitor binding affinity and potency. Azaindazole 2 (Table 1 ) in which a polar nitrogen has been incorporated into the indazole back pocket served the purpose of reducing overall lipophilicity relative to GSK583 but at the expense of inhibitor potency in both the fluorescence polarization (FP) and whole blood assay formats. Despite the reduction in lipophilicity, this change did not result in any appreciable reduction in potency of 2 against the hERG ion channel relative to GSK583. The reduction in potency of $\mathbf{2}$ is likely due to disruption of the interaction with Glu66 in the back pocket of the RIP2 protein. Building back lipophilicity without the aromaticity provided the tetrahydroindazole 3, which also led to reduced activity at RIP2 kinase while maintaining activity against the hERG ion channel. Complete removal of the aromatic ring of the indazole gave pyrazole 4, which maintained the energetically favorable hydrogen bonding interaction with Asp164 of RIP2 while significantly reducing the overall lipophilicity of the molecule. However, the reduced potency of 4 could again be attributed to a loss of the stabilizing interaction with Glu66. Surprisingly, this modification also maintained potency versus the hERG channel despite the loss of aromatic character and a significant reduction in overall lipophilicity. Incorporation of a methyl group on position 5 of the pyrazole back pocket moiety of 5 resulted in only 
Table 1. Focused Back Pocket Modifications of GSK583

(23)

a small increase in potency in the FP binding assay format relative to 4 . While small alkyl substituents at position 5 were unlikely to maintain the interaction with Glu66 as the aliphatic $\mathrm{C}-\mathrm{H}$ is less polar and further removed from Glu66 than that of GSK583, small alkyl substituents at the 4-position of the pyrazole were expected to occupy hydrophobic space in the back pocket. Gratifyingly, dimethylpyrazole 6 possessed good potency in both the FP and whole blood assays as well as decreased activity at the hERG channel. ${ }^{21}$ The steric interactions imparted by the methyl groups in combination with the overall reduction in the lipophilicity of 6 is likely responsible for the reduced affinity for the hERG channel. In combination, this structural modification has increased the window to hERG activity approximately 5-fold relative to GSK583 with a 155-fold overall window between the human whole blood potency and hERG $\mathrm{IC}_{50}$. The increased activity in the whole blood assay format combined with the reduced lipophilicity also resulted in an overall increase in ligand binding efficiency as seen with an increased LipE relative to GSK583.

The iv and po pharmacokinetics of dimethylpyrazole 6 in the rat are summarized in Table 2. Following i.v. administration, 6 exhibited low clearance in the rat with a moderate volume of distribution and a half-life of approximately $4 \mathrm{~h}$. Upon oral administration, 6 had an oral bioavailability of $82 \%$. In comparison with GSK583, the dimethylpyrazole 6 showed an overall improved pharmacokinetic profile with a higher plasma free fraction, both key components for improved inhibitor efficacy and lower predicted human dose.

Although the overall pharmacokinetic profile and selectivity versus the hERG channel of dimethylpyrazole 6 was improved versus GSK583, a further increase of inhibitor potency would serve to potentially widen this critical window, which was required for further progression of this series of inhibitors. As with most ATP-competitive kinase inhibitors, the hinge binding interaction of the inhibitor with the protein is a key component in determining inhibitor potency. To improve upon the potency of the 4-aminoquinoline series of RIP2 kinase inhibitors, a strategy to further strengthen this critical interaction was employed. As shown in Figure 1, the X-ray co-crystal structure of GSK583 bound within the ATP binding pocket of RIP2 kinase revealed the single point hinge binding interaction between $\mathrm{N} 1$ of the 4aminoquinoline core and Met98 of the protein. In order to strengthen this interaction, substitution of the 4-aminoquinoline core with electron donating groups that would serve to increase the hinge binding ability were explored. ${ }^{22}$ Examination of the Xray co-crystal structure suggested that the incorporation of electron donating groups at the $\mathrm{C} 7$ position of the 4aminoquinoline template would be best tolerated as this position is oriented away from the protein toward the solvent front. ${ }^{6}$ As shown in Table 3, inhibitor 7, which incorporates the C7 methoxy group, resulted in a 9-fold increase in whole blood potency relative to $6 .{ }^{23}$ While this modification resulted in a small increase in activity versus the hERG ion channel, which can be attributed to the increased basicity of the $\mathrm{N} 1$ quinoline nitrogen, the increased potency versus RIP2 kinase led to the desired greater than 100-fold window between the human whole blood potency and inhibition of the hERG channel. A variety of modifications at the $\mathrm{C} 7$ position of the 4-aminoquinoline core was tolerated in terms of binding activity in the fluorescence polarization assay format (Table 3 ). However, small groups that had the ability to donate electron density through resonance had improvements in cellular activity relative to 6 . Compounds with electron withdrawing substituents (11) or with substituents that are moderately electron donating through inductive effects (12) did not give improved cell based activity as observed in compounds 7-10. While compounds 9 and 10 were potent in the human whole blood assay and lacked activity against the hERG ion channel, the added polarity introduced by these groups were seen to limit the oral pharmacokinetics (data not shown) of these inhibitors thereby limiting their further progression.

Patch clamp electrophysiology (QPatch; Sophion Bioscience) has been regarded as the gold standard assay for assessing ion channel modulation and the propensity of new chemical entities to elicit QT prolongation in humans. Although the increase in selectivity versus the hERG channel in this assay format was encouraging, further evaluation of the proarrhythmic potential beyond the QPatch analysis of 7 was warranted. It has been well established that the hERG patch clamp assay, in isolation, does not provide the level of predictivity required to fully discharge the risk of a torsade de pointes (TdP) arrhythmia. In order to more accurately assess these risks in a preclinical in vitro assay, the proarrhythmic potential of inhibitor 7 was evaluated in the arterially perfused rabbit left ventricular wedge model. ${ }^{24}$ In this assay, four major readouts, QT prolongation (a measure of ventricular repolarization), transmural dispersion of repolarization (Tp-e interval), impulse conduction (QRS interval), and isometric contractile force (ICF), are measured to allow for a more in depth, nuanced understanding of the proarrhythmic potential of new chemical entities. Further, a TdP score derived from QT, Tp-e, and observations of early afterdepolarizations

Table 2. Pharmacokinetic Profiles of GSK583 and 6 in the Sprague-Dawley Rat

$\begin{array}{lcccccccc}\text { Cmpd } & \text { IV/oral dose }(\mathrm{mg} / \mathrm{kg}) & \mathrm{Cl}(\mathrm{mL} / \mathrm{min} / \mathrm{kg}) & \mathrm{Vdss}(\mathrm{L} / \mathrm{kg}) & \mathrm{IV} T_{1 / 2}(\mathrm{~h}) & \text { oral } C_{\max }(\mathrm{ng} / \mathrm{mL}) & \text { oral AUC }(\mathrm{ng} \cdot \mathrm{h} / \mathrm{mL}) & \% \mathrm{~F} & \text { free fraction }(\%) \\ \text { GSK583 } & 1.0 / 2.0 & 15 & 2.0 & 7.0 & 260 & 980 & 39 & 2.6 \\ 6 & 2.0 / 2.0 & 12 & 2.1 & 4.2 & 990 & 3800 & 82 & 11.2\end{array}$


Table 3. Modification of the C7 Position of Inhibitor 6

\begin{tabular}{|c|c|c|c|c|c|c|c|}
\hline Cmpd & $\mathrm{R}$ & RIPK2 FP IC $50(n M)$ & RIPK2 $\mathrm{hWB} \mathrm{IC}_{50}(\mathrm{nM})$ & $\operatorname{cLog} \mathrm{P}$ & LipE & $\mathrm{hERG} \mathrm{IC}_{50}(\mathrm{nM})$ & hERG/hWB fold shift \\
\hline 6 & $\mathrm{H}$ & 6.3 & 100 & 3.3 & 4.9 & 15500 & 155 \\
\hline 7 & $\mathrm{OCH}_{3}$ & 1.3 & 11 & 3.0 & 5.9 & 14500 & 1318 \\
\hline 8 & $\mathrm{OCH}_{2} \mathrm{CH}_{3}$ & 1.6 & 41 & 3.5 & 5.3 & $>30000$ & $>700$ \\
\hline 9 & $\mathrm{OCH}_{2} \mathrm{CH}_{2} \mathrm{OH}$ & 2.5 & 15 & 2.1 & 6.5 & $>30000$ & $>2000$ \\
\hline 10 & $\mathrm{OCH}_{2} \mathrm{CH}_{2} \mathrm{OMe}$ & 2.5 & 5.0 & 2.8 & 5.8 & 30000 & 6000 \\
\hline 11 & $\mathrm{OCF}_{3}$ & 6.3 & 446 & 4.1 & 4.1 & $>30000$ & $>100$ \\
\hline 12 & Et & 2.5 & 154 & 4.3 & 4.3 & 23990 & 156 \\
\hline
\end{tabular}

Table 4. Summary Data for Inhibitor 7 in Rabbit Cardiac Wedge Preparation (Mean \pm SEM)

\begin{tabular}{|c|c|c|c|c|c|c|}
\hline & control & $0.3 \mu \mathrm{M}$ & $1 \mu \mathrm{M}$ & $3 \mu \mathrm{M}$ & $10 \mu \mathrm{M}$ & desired range \\
\hline $\mathrm{QT}(\mathrm{ms})$ & $353.6 \pm 10.5$ & $360.6 \pm 10.8$ & $366.3 \pm 12.2$ & $376.1 \pm 10.2$ & $386.6 \pm 14.5$ & $\Delta<10 \%$ \\
\hline$\Delta \mathrm{QT} \%$ & & $2.0 \pm 1.0$ & $3.6 \pm 1.5$ & $6.4 \pm 1.7$ & $9.3 \pm 1.7$ & \\
\hline Tp-e (ms) & $59.2 \pm 4.8$ & $64.2 \pm 5.3$ & $67.9 \pm 6.6$ & $73.3 \pm 8.0$ & $77.0 \pm 10.8$ & NA \\
\hline $\mathrm{QRS}(\mathrm{ms})$ & $33.9 \pm 2.7$ & $34.9 \pm 2.8$ & $35.4 \pm 2.8$ & $35.2 \pm 2.4$ & $36.3 \pm 1.6$ & $\Delta<10 \%$ \\
\hline ICF (\% change) & 0 & $-9.9 \pm 2.1$ & $-17.3 \pm 2.8$ & $-22.6 \pm 3.4$ & $-28.0 \pm 4.5$ & $\Delta<50 \%$ \\
\hline TdP score & 0 & 0.25 & 0.75 & 1.25 & 1.5 & $<2.5$ \\
\hline
\end{tabular}

Table 5. PK Parameters for Compound 7 in Rat and Dog, Discrete iv/po Studies, and Predicted Human PK

\begin{tabular}{|c|c|c|c|c|c|c|c|c|}
\hline species & IV/oral dose ( $\mathrm{mg} / \mathrm{kg})$ & $\mathrm{Cl}(\mathrm{mL} / \mathrm{min} / \mathrm{kg})$ & Vdss (L/kg) & $\operatorname{IV~} T_{1 / 2}(\mathrm{~h})$ & oral $C_{\max }(\mathrm{ng} / \mathrm{mL})$ & oral AUC (ng.h/mL) & $\% \mathrm{~F}$ & free fraction (\%) \\
\hline rat & $1.1 / 2.0$ & 12 & 1.1 & 3.1 & 740 & 2400 & 85 & 5.5 \\
\hline $\operatorname{dog}$ & $1.1 / 2.0$ & 20 & 3.5 & 2.5 & 330 & 1900 & 96 & 16.3 \\
\hline human (predicted) & $1.5^{a}$ & 6.8 & 1.9 & 6.2 & 259 & 2510 & 74 & 9.6 \\
\hline
\end{tabular}

(ectopic beating) is calculated to quantify the torsadogenic potential of drugs. The overall effects of 7 with respect to these cardiac parameters over a concentration range of 0.3 to $10 \mu \mathrm{M}$ relative to control are summarized in Table 4. Here, inhibitor 7 produced a concentration-dependent prolongation of $\mathrm{QT}$ interval up to $9.3 \%$ at a concentration of $10 \mu \mathrm{M}$. While Tp-e also increased in a concentration-dependent manner, TdP scores generated from the QT, Tp-e, and the absence of early afterdepolarizations did not exceed 1.5 at the top dose of 10 $\mu \mathrm{M}$. A TdP score of $\geq 2.5$ has been observed for compounds associated with clinical torsadogenic potential. ${ }^{24}$ In addition, the effect on QRS was $<10 \%$, suggesting a lack of issues related to impulse conduction, while the observed decreases in contractility fall in line with the expected inherent decrease in contractility over time in the rabbit ventricular wedge preparation. The industry accepted standard for a safety window between hERG $\mathrm{IC}_{50}$ and therapeutic free $C_{\max }$ plasma concentrations has been previously reported by Redfern. ${ }^{25}$ A $30-100$-fold window is regarded as acceptable when taking disease severity and medical need into account. Given the observation of a 9.3\% QT prolongation at $10 \mu \mathrm{M}$ in a rabbit left ventricular wedge model for inhibitor 7 as well as an $\mathrm{IC}_{50}$ of $14.5 \mu \mathrm{M}$ in the hERG QPatch assay, a maximum free $C_{\max }$ of $100 \mathrm{nM}(38.8 \mathrm{ng} / \mathrm{mL})$ would be required to provide a 100 -fold window between free drug concentration and hERG related activities that could lead to QT prolongation and increased potential for torsades de pointes arrhythmias.

To place this improvement in selectivity versus the hERG channel in the proper therapeutically relevant context, a prediction of the human pharmacokinetics was conducted.
Evaluation of the pharmacokinetics of analog 7 (Table 5) showed that C7 substitution had little effect on the pharmacokinetic profile relative to 6 (Table 2). Analog 7 demonstrated low clearance and volume of distribution in the rat with an oral bioavailability of $85 \%$ following solution dosing. In the dog, 7 is a moderately cleared molecule with a moderately large volume of distribution and an oral bioavailability of $96 \%$. These pharmacokinetic parameters were utilized in an in silico model to predict the human pharmacokinetic profile of inhibitor 7 (Table 5). Based upon the predicted human PK profile and the potency of inhibitor 7 in the human whole blood assay $\left(\mathrm{IC}_{50}=10\right.$ nM; Table 3), it was determined that a $54 \mathrm{mg}$ twice daily dose (b.i.d.) would be required to inhibit RIP2 kinase at an average $90 \%$ level for a period of $24 \mathrm{~h}$. At this dosing interval, a predicted plasma $C_{\max }$ of $259 \mathrm{ng} / \mathrm{mL}(666 \mathrm{nM})$ was estimated. Taking into account the human free fraction of $9.6 \%$ (Table 5), the free plasma $C_{\max }$ concentration would be predicted to be $24.8 \mathrm{ng} / \mathrm{mL}$ $(64 \mathrm{nM})$. This is well below the $10 \mu \mathrm{M}$ free $C_{\max }$ determined in the rabbit left ventricular wedge required to trigger a prolongation of the $\mathrm{QT}$ interval and potentially lethal arrhythmias (156-fold shift) and the $14.5 \mu \mathrm{M}$ activity at the hERG ion channel (227-fold shift).

In addition to selectivity versus the hERG ion channel inhibitor, 7 also possessed broad kinase selectivity when tested against a panel of 299 kinases (see Supporting Information). In total, 15 of the kinases in the panel were inhibited $>70 \%$ at a concentration of $1 \mu \mathrm{M}$ of inhibitor 7 . Not unexpectedly off-target kinase interactions were predominantly observed to be within the tyrosine kinase subfamily of kinases stemming from either the Src family kinase or Ephrin receptor subfamily with the exceptions of 
ABL2 and ZAK kinases, which are inhibited at levels of $81 \%$ and $89 \%$ inhibition, respectively. BRK, FRK, c-SRC, EPHB4, and TXK are all inhibited at levels $>90 \%$ inhibition.

The X-ray co-crystal structure of 7 bound within the ATP binding pocket of RIP2 kinase (Figure 3 ) revealed it to bind in the

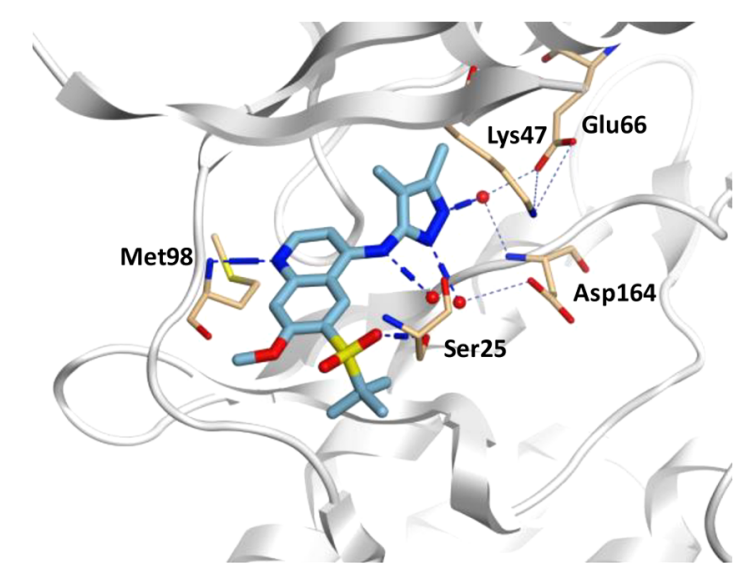

Figure 3. X-ray co-crystal structure of 7 bound within the ATP binding pocket of RIP2 kinase $(2.53 \AA)$.

manner of a type I kinase inhibitor with the hinge interaction with Met98 through the quinoline nitrogen, sulfone interaction with the Ser25 in the glycine rich loop, and interaction with the Asp164 of the DFG loop through two water molecules. Each of these interactions is important for activity as determined through our previous SAR studies. ${ }^{6}$

To establish a PK/PD relationship of inhibitor 7 and compare it with the efficacy of GSK583, dimethylpyrazole 7 was evaluated in vivo in the acute MDP-driven rat peritonitis model, which has been previously detailed. ${ }^{6}$ Following oral administration of inhibitor 7, the reduction of cytokine levels at $2 \mathrm{~h}$ for each dose group was used to generate an in vivo $\mathrm{IC}_{50}$ value of $19 \mathrm{nM}(7.5$ $\mathrm{ng} / \mathrm{mL}$ ) (Figure 4). This value is in good agreement with the
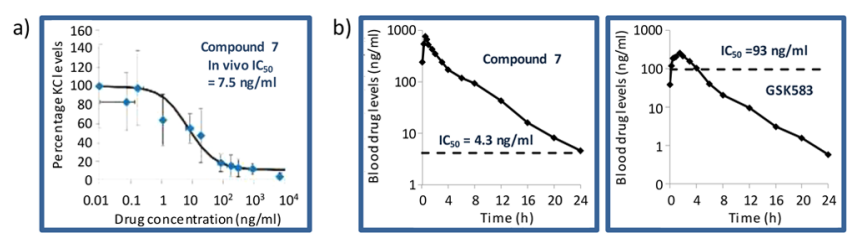

Figure 4. (a) Concentration-response plot for inhibitor 7 at a single time point $(2 \mathrm{~h}$ ) over ten doses. (b) Comparison of human whole blood $\mathrm{IC}_{50}$ values and drug levels of GSK583 and compound 7.

measured rat in vitro whole blood $\mathrm{IC}_{50}$ value of $13 \mathrm{nM}(5.0 \mathrm{ng} /$ $\mathrm{mL}$ ), which in turn is in close agreement with the human whole blood value of $11 \mathrm{nM}(4.3 \mathrm{ng} / \mathrm{mL})$, indicative of coherent pharmacology across species in vitro and a good correlation between in vitro and in vivo assays in rats. Putting the human whole blood $\mathrm{IC}_{50}$ value into context with the drug levels in rat following a $2 \mathrm{mg} / \mathrm{kg}$ oral dose allows for a direct comparison of tool GSK583 and compound 7. Observed blood levels of compound 7 are above its human whole blood $\mathrm{IC}_{50}$ over a period of $24 \mathrm{~h}$ while GSK583 blood concentrations fall below its corresponding human whole blood $\mathrm{IC}_{50}$ after only $4 \mathrm{~h}$. This comparison at comparable doses indicates that much lower doses of compound 7 would be needed to achieve targeted inhibition levels over a $24 \mathrm{~h}$ period as compared to GSK583, and thus, a more suitable clinical candidate for a novel target where levels of inhibition needed to have an effect on inflammatory diseases have not yet been defined.

We have detailed previously the discovery of the potent and kinome-selective RIP2 kinase inhibitor GSK583. Despite GSK583's positive attributes, it was not progressed to more advanced safety studies due to a combination of limiting properties: potent interaction with the hERG ion channel, less than optimal pharmacokinetics, as well as subpar efficacy in an in vivo model of inflammation. In this Letter we have detailed efforts to modify the GSK583 template to a progressable compound to be evaluated in advanced safety studies. The cornerstone of the strategy to identify a more suitable inhibitor for advancement was to reduce the overall lipophilicity of GSK583, which was identified as a key contributor to the lack of selectivity versus the hERG channel as well as the poor pharmacokinetics and overall in vivo efficacy. Toward this end, structure-enabled design suggested elimination of the aromatic ring of the back pocket benzimidazole and introduction of a C7 methoxy group leading to the potent and selective RIP2 kinase inhibitor 7. These efforts have resulted in an overall increase in potency in the human whole blood assay, reduced affinity for the hERG channel, significantly improved pharmacokinetics in preclinical species, greater efficacy in an in vivo model of inflammation, and a reasonable predicted human dose. Taken together, these improvements in both the physical properties of inhibitor 7 as well as this inhibitor's overall efficacy now warrant its evaluation in more advanced safety studies.

\section{ASSOCIATED CONTENT}

\section{S Supporting Information}

The Supporting Information is available free of charge on the ACS Publications website at DOI: 10.1021/acsmedchemlett.8b00344.

Experimental details and characterization of compounds 2-12; biological assay details; kinome selectivity data for compound 7; data collection and refinement statistics and exemplars of the density for compound 7 (PDF)

\section{Accession Codes}

The PDB codes are as follows: 6HMX for RIP2/7 co-crystal structure.

\section{AUTHOR INFORMATION}

\section{Corresponding Author}

*E-mail: pamela.a.haile@gsk.com.

ORCID $\odot$

Pamela A. Haile: 0000-0003-3950-4890

\section{Notes}

The authors declare the following competing financial interest(s): All GSK authors are/were employees and stockholders of GlaxoSmithKline when this work was completed.

\section{ACKNOWLEDGMENTS}

The authors would like to express appreciation to Emma Jones, Phil Hardwicke, and Robert Leydon for protein production, Julie Cox for screening support, Ashley Leister and Amy Gibble for purification support, and Allison Beal for review.

\section{ABBREVIATIONS}

NOD, nucleotide-binding oligomerization domain protein; PRR, pattern recognition receptors; RIP2, receptor interacting 
protein 2; IBD, inflammatory bowel disease; FP, fluorescence polarization; clogP, calculated partition coefficient; MW, molecular weight; LipE, lipophilic ligand efficiency; ie-DAP, GD-glutamyl-meso-diaminopimelic acid; MDP, muramyl dipetide; TLR, toll-like receptor; hERG, human ether-a-go-go-related gene

\section{REFERENCES}

(1) Gatheral, T.; Reed, D. M.; Moreno, L.; Gough, P. J.; Votta, B. J.; Sehon, C. A.; Rickard, D. J.; Bertin, J.; Lim, E.; Nicholson, A. G.; Mitchell, J. A. A key role for the endothelium in NOD1 mediated vascular inflammation: comparison to TLR4 responses. PLoS One 2012, 7, e42386.

(2) Moreno, L.; Gatheral, T. Therapeutic targeting of NOD1 receptors. Br. J. Pharmacol. 2013, 170, 475-485.

(3) Schirbel, A.; Kessler, S.; Rieder, F.; West, G.; Rebert, N.; Asosingh, K.; McDonald, C.; Fiocchi, C. Pro-Angiogenic Activity of TLRs and NLRs: A Novel Link Between Gut Microbiota and Intestinal Angiogenesis. Gastroenterology 2013, 144, 613-623.

(4) Sfriso, P.; Caso, F.; Tognon, S.; Galozzi, P.; Gava, A.; Punzi, L. Blau syndrome, clinical and genetic aspects. Autoimmun. Rev. 2012, 12, 4451.

(5) Hugot, J. P.; Chamaillard, M.; Zouali, H.; Lesage, S.; Cezard, J. P.; Belaiche, J.; Almer, S.; Tysk, C.; O’Morain, C. A.; Gassull, M.; Binder, V.; Finkel, Y.; Cortot, A.; Modigliani, R.; Laurent-Puig, P.; GowerRousseau, C.; Macry, J.; Colombel, J. F.; Sahbatou, M.; Thomas, G. Association of NOD2 leucine-rich repeat variants with susceptibility to Crohn's disease. Nature 2001, 411 (6837), 599-603.

(6) Haile, P. A.; Votta, B. J.; Marquis, R. W.; Bury, M. J.; Mehlmann, J. F.; Singhaus, R., Jr.; Charnley, A. K.; Lakdawala, A. S.; Convery, M. A.; Lipshutz, D. B.; Desai, B. M.; Swift, B.; Capriotti, C. A.; Berger, S. B.; Mahajan, M. K.; Reilly, M. A.; Rivera, E. J.; Sun, H. H.; Nagilla, R.; Beal, A. M.; Finger, J. N.; Cook, M. N.; King, B. W.; Ouellette, M. T.; Totoritis, R. D.; Pierdomenico, M.; Negroni, A.; Stronati, L.; Cucchiara, S.; Ziolkowski, B.; Vossenkamper, A.; MacDonald, T. T.; Gough, P. J.; Bertin, J.; Casillas, L. N. The Identification and Pharmacological Characterization of 6-(tert-Butylsulfonyl)-N-(5-fluoro-1H-indazol-3yl)quinolin-4-amine (GSK583), a Highly Potent and Selective Inhibitor of RIP2 Kinase. J. Med. Chem. 2016, 59, 4867-4880.

(7) Tigno-Aranjuez, J. T.; Benderitter, P.; Rombouts, F.; Deroose, F.; Bai, X.; Mattioli, B.; Cominelli, F.; Pizarro, T. T.; Hoflack, J.; Abbott, D. W. In vivo inhibition of RIPK2 kinase alleviates inflammatory disease. J. Biol. Chem. 2014, 289, 29651-29664.

(8) Canning, P.; Ruan, Q.; Schwerd, T.; Hrdinka, M.; Maki, J. L.; Saleh, D.; Suebsuwong, C.; Ray, S.; Brennan, P. E.; Cuny, G. D.; Uhlig, H. H.; Gyrd-Hansen, M.; Degterev, A.; Bullock, A. N. Inflammatory Signaling by NOD-RIPK2 Is Inhibited by Clinically Relevant Type II Kinase Inhibitors. Chem. Biol. 2015, 22, 1174-1184.

(9) Nachbur, U.; Stafford, C. A.; Bankovacki, A.; Zhan, Y.; Lindqvist, L. M.; Fiil, B. K.; Khakham, Y.; Ko, H. J.; Sandow, J. J.; Falk, H.; Holien, J. K.; Chau, D.; Hildebrand, J.; Vince, J. E.; Sharp, P. P.; Webb, A. I.; Jackman, K. A.; Muhlen, S.; Kennedy, C. L.; Lowes, K. N.; Murphy, J. M.; Gyrd-Hansen, M.; Parker, M. W.; Hartland, E. L.; Lew, A. M.; Huang, D. C.; Lessene, G.; Silke, J. A RIPK2 inhibitor delays NOD signalling events yet prevents inflammatory cytokine production. Nat. Commun. 2015, 6, $6442-6454$.

(10) He, X.; Da Ros, S.; Nelson, J.; Zhu, X.-F.; Jiang, T.; Okram, B.; Jiang, S.; Michellys, P.-Y.; Iskandar, M.; Espinola, S.; Jia, Y.; Bursulaya, B.; Kreusch, A.; Gao, M.-Y.; Spraggon, G.; Baaten, J. E.; Clemmer, L.; Meeusen, S.; Huang, D.; Hill, R.; Nguyen-Tran, V.; Fathman, J.; Liu, B.; Tuntland, T.; Gordon, W. P.; Hollenbeck, T.; Ng, K.; Shi, J.; Bordone, L.; Liu, H. Identification of Potent and Selective RIPK2 Inhibitors for the Treatment of Inflammatory Diseases. ACS Med. Chem. Lett. 2017, 8, $1048-1053$.

(11) Goncharov, T.; Hedayati, S.; Mulvihill, M. M.; Izrael-Tomasevic, A.; Zobel, K.; Jeet, S.; Fedorova, A. V.; Eidenschenk, C.; deVoss, J.; Yu, K.; Shaw, A. S.; Kirkpatrick, D. S.; Fairbrother, W. J.; Deshayes, K.;
Vucic, D. Disruption of XIAP-RIP2 Association Blocks NOD2Mediated Inflammatory Signaling. Mol. Cell 2018, 69, 551-565.

(12) van Heel, D. A.; Ghosh, S.; Butler, M.; Hunt, K.; Foxwell, B. M.; Mengin-Lecreulx, D.; Playford, R. J. Synergistic enhancement of Tolllike receptor responses by NOD1 activation. Eur. J. Immunol. 2005, 35, 2471-2476.

(13) Fritz, J. H.; Girardin, S. E.; Fitting, C.; Werts, C.; MenginLecreulx, D.; Caroff, M.; Cavaillon, J. M.; Philpott, D. J.; Adib-Conquy, M. Synergistic stimulation of human monocytes and dendritic cells by Toll-like receptor 4 and NOD1- and NOD2-activating agonists. Eur. J. Immunol. 2005, 35, 2459-2470.

(14) Jakopin, Ž. Nucleotide-Binding Oligomerization Domain (NOD) Inhibitors: A Rational Approach toward Inhibition of NOD Signaling Pathway. J. Med. Chem. 2014, 57, 6897-6918.

(15) Karaman, M. W.; Herrgard, S.; Treiber, D. K.; Gallant, P.; Atteridge, C. E.; Campbell, B. T.; Chan, K. W.; Ciceri, P.; Davis, M. I.; Edeen, P. T.; Faraoni, R.; Floyd, M.; Hunt, J. P.; Lockhart, D. J.; Milanov, Z. V.; Morrison, M. J.; Pallares, G.; Patel, H. K.; Pritchard, S.; Wodicka, L. M.; Zarrinkar, P. P. A quantitative analysis of kinase inhibitor selectivity. Nat. Biotechnol. 2008, 26, 127-132.

(16) Adams, J. L.; Gallagher, T. F.; Lee, J. C.; White, J. R. Imidazole derivatives and their use as cytokine inhibitors. Patent WO9314081A1, 1993.

(17) Joshi, A.; Dimino, T.; Vohra, Y.; Cui, C.; Yan, G.-X. Preclinical strategies to assess QT liability and torsadogenic potential of new drugs: The role of experimental models. Journal of Electrocardiology 2004, 37, $7-14$.

(18) Jamieson, C.; Moir, E. M.; Rankovic, Z.; Wishart, G. Medicinal Chemistry of hERG Optimizations: Highlights and Hang-Ups. J. Med. Chem. 2006, 49, 5029-5046.

(19) Cavalli, A.; Poluzzi, E.; De Ponti, F.; Recanatini, M. Toward a Pharmacophore for Drugs Inducing the Long QT Syndrome: Insights from a CoMFA Study of HERG K+ Channel Blockers. J. Med. Chem. 2002, 45, 3844-3853.

(20) Horowitz, S.; Trievel, R. C. Carbon-Oxygen Hydrogen Bonding in Biological Structure and Function. J. Biol. Chem. 2012, 287, 4157641582.

(21) Casillas, L. N.; Charnley, A. K.; Haile, P. A.; Hughes, T. V.; Marquis, R. W.; Mehlmann, J. F.; Reilly, M.; Romano, J. J.; Singhaus, R. $\mathrm{R}$. Preparation of quinolylamine derivatives for use as kinase inhibitors. WO2012021580A1, 2012.

(22) Potashman, M. H.; Bready, J.; Coxon, A.; DeMelfi, T. M.; DiPietro, L.; Doerr, M.; Elbaum, D.; Estrada, J.; Gallant, P.; Germain, J.; Gu, Y.; Harmange, J.-C.; Kaufman, S. A.; Kendall, R.; Kim, J. L.; Kumar, G. N.; Long, A. M.; Neervannan, S.; Patel, V. F.; Polverino, A.; Rose, P.; van der Plas, S.; Whittington, D.; Zanon, R.; Zhao, H. Design, Synthesis, and Evaluation of Orally Active Benzimidazoles and Benzoxazoles as Vascular Endothelial Growth Factor-2 Receptor Tyrosine Kinase Inhibitors. J. Med. Chem. 2007, 50, 4351-4373.

(23) Bury, M. J.; Casillas, L. N.; Charnley, A. K.; Haile, P. A.; Marquis, R. W.; Mehlmann, J. F.; Romano, J. J.; Singhaus, R. R., Wang, G. Z. Preparation of amino-quinolines as RIP2 kinase inhibitors. WO2012122011A2, 2012.

(24) Liu, T.; Brown, B. S.; Wu, Y.; Antzelevitch, C.; Kowey, P. R.; Yan, G.-X. Blinded validation of the isolated arterially perfused rabbit ventricular wedge in preclinical assessment of drug-induced proarrhythmias. Heart rhythm: the official journal of the Heart Rhythm Society 2006, 3, 948-956.

(25) Redfern, W. S.; Carlsson, L.; Davis, A. S.; Lynch, W. G.; MacKenzie, I.; Palethorpe, S.; Siegl, P. K. S.; Strang, I.; Sullivan, A. T.; Wallis, R.; Camm, A. J.; Hammond, T. G. Relationships between preclinical cardiac electrophysiology, clinical QT interval prolongation and torsade de pointes for a broad range of drugs: evidence for a provisional safety margin in drug development. Cardiovasc. Res. 2003, $58,32-45$. 